\title{
Long-term albumin administration in decompensated cirrhosis (ANSWER): an open-label randomised trial
}

\author{
Paolo Caraceni, Oliviero Riggio, Paolo Angeli, Carlo Alessandria, Sergio Neri, Francesco G Foschi, Fabio Levantesi, Aldo Airoldi, Sergio Boccia, \\ Gianluca Svegliati-Baroni, Stefano Fagivoli, Roberto G Romanelli, Raffaele Cozzolongo, Vito Di Marco, Vincenzo Sangiovanni, \\ Filomena Morisco, Pierluigi Toniutto, Annalisa Tortora, Rosanna De Marco, Mario Angelico, Irene Cacciola, Gianfranco Elia, \\ Alessandro Federico, Sara Massironi, Riccardo Guarisco, Alessandra Galioto, Giorgio Ballardini, Maria Rendina, Silvia Nardelli, Salvatore Piano, \\ Chiara Elia, Loredana Prestianni, Federica Mirici Cappa, Lucia Cesarini, Loredana Simone, Chiara Pasquale, Marta Cavallin, Alida Andrealli, \\ Federica Fidone, Matteo Ruggeri, Andrea Roncadori, Maurizio Baldassarre, Manuel Tufoni, Giacomo Zaccherini, Mauro Bernardi, for the \\ ANSWER Study Investigators*
}

\section{Summary}

Background Evidence is scarce on the efficacy of long-term human albumin (HA) administration in patients with decompensated cirrhosis. The human Albumin for the treatmeNt of aScites in patients With hEpatic ciRrhosis (ANSWER) study was designed to clarify this issue.

Methods We did an investigator-initiated multicentre randomised, parallel, open-label, pragmatic trial in 33 academic and non-academic Italian hospitals. We randomly assigned patients with cirrhosis and uncomplicated ascites who were treated with anti-aldosteronic drugs $(\geq 200 \mathrm{mg} /$ day) and furosemide ( $\geq 25 \mathrm{mg} /$ day) to receive either standard medical treatment (SMT) or SMT plus HA (40 g twice weekly for 2 weeks, and then $40 \mathrm{~g}$ weekly) for up to 18 months. The primary endpoint was 18-month mortality, evaluated as difference of events and analysis of survival time in patients included in the modified intention-to-treat and per-protocol populations. This study is registered with EudraCT, number 2008-000625-19, and ClinicalTrials.gov, number NCT01288794.

Findings From April 2, 2011, to May 27, 2015, 440 patients were randomly assigned and 431 were included in the modified intention-to-treat analysis. 38 of 218 patients died in the SMT plus HA group and 46 of 213 in the SMT group. Overall 18-month survival was significantly higher in the SMT plus HA than in the SMT group (Kaplan-Meier estimates $77 \%$ vs $66 \% ; \mathrm{p}=0.028)$, resulting in a $38 \%$ reduction in the mortality hazard ratio $(0 \cdot 62$ [95\% CI $0 \cdot 40-0 \cdot 95])$. $46(22 \%)$ patients in the SMT group and $49(22 \%)$ in the SMT plus HA group had grade 3-4 non-liver related adverse events.

Interpretation In this trial, long-term HA administration prolongs overall survival and might act as a disease modifying treatment in patients with decompensated cirrhosis.

Funding Italian Medicine Agency.

Copyright (C) 2018 Elsevier Ltd. All rights reserved.

\section{Introduction}

Cirrhosis of the liver is a leading cause of disability and mortality worldwide. ${ }^{1,2}$ In Europe, 170000 individuals die from cirrhosis every year, accounting for $1.8 \%$ of all deaths, and a similar proportion has been reported from the USA. ${ }^{3,4}$ Complications, such as ascites, gastrointestinal bleeding, hepatic encephalopathy, and jaundice, herald the decompensation of cirrhosis - the final stage of the disease. $^{5}$ Decompensated cirrhosis carries a poor prognosis because the median survival time is about 2 years, and it imposes a heavy burden on health-care costs, mainly due to the need for repeated hospital admissions. ${ }^{5,6}$

The current approach to patients with decompensated cirrhosis relies on the individual management of each complication. Therefore, there is an unmet need for an overall therapeutic strategy able to prevent the development of complications, thus reducing hospital readmissions and costs, and improving quality of life and survival.

Long-term human albumin (HA) administration to patients with ascites, the most common cause of decompensation, ${ }^{7}$ has been debated for decades. Because reduced serum albumin concentration is commonly seen in patients with advanced cirrhosis, a putative rationale underlying HA use relies on the belief that an improvement in hypoalbuminaemia would slow down ascites formation by increasing plasma colloid-osmotic pressure. However, hypoalbuminaemia per se does not have a pre-eminent pathogenetic role in this process because the colloid-osmotic pressure gradient, a factor regulating fluid partition between plasma and the interstitial space, is not reduced in cirrhosis with ascites. Moreover, normalisation of serum colloid-osmotic pressure achieved by repeated HA administrations did not ensure a persistent control of ascites once

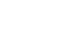

Lancet 2018; 391: 2417-29 Published Online May 31, 2018 http://dx.doi.org/10.1016/ S0140-6736(18)30840-7

This online publication has been corrected. The corrected version first appeared at thelancet.com on August 2, 2018

See Comment page 2391

*Investigators listed at the end of the paper

Department of Medical and Surgical Sciences, and Center for Applied Biomedical Research, University of Bologna, Bologna, Italy (P Caraceni MD,

$M$ Baldassarre PhD, M Tufoni MD, G Zaccherini MD, Prof M Bernardi MD); Department of Clinical Medicine, Sapienza University of Rome, Rome, Italy (O Riggio MD, S Nardelli MD, ( Pasquale MD); Unit of Internal Medicine and Hepatology, Department of Medicine, University of Padua, Padua Italy (Prof P Angeli PhD S Piano PhD, M Cavallin MD); Division of Gastroenterology and Hepatology, Città della Salute e della Scienza Hospital, University of Turin, Turin, Italy (CAlessandria MD, C Elia MD, A Andrealli MD); Department of Clinical and Experimental Medicine, University of Catania, Catania, Italy (S Neri MD, L Prestianni MD, F Fidone MD); Internal Medicine, Hospital of Faenza, Azienda Unità Sanitaria Locale of Romagna, Faenza, Italy (F G Foschi MD, F M Cappa PhD); Internal Medicine, Hospital of Bentivoglio, AUSL of Bologna, Bologna, Italy (F Levantesi MD); Liver Unit, Department of Hepatology and Gastroenterology, Niguarda Hospital, Milan, Italy 
(A Airoldi MD, LCesarini MD); Gastroenterology Unit, University Hospital, Ferrara, Italy (S Boccia MD, L Simone MD); Department of Gastroenterology, Polytechnic University of Marche, Ancona, Italy (G Svegliati-Baroni MD); Gastroenterology and Transplant Hepatology, Papa Giovanni XXIII Hospital, Bergamo, Italy (S Fagiuoli MD); Department of Experimental and Clinical Medicine, University of Florence, Florence, Italy

(R G Romanelli PhD); Division of

Gastroenterology, National Institute of Gastroenterology S De Bellis, Castellana Grotte (Bari), Italy (R Cozzolongo MD); Unit of Gastroenterology and Hepatology, Biomedical Department of Internal and Specialistic Medicine, University of Palermo, Palermo, Italy

(Prof V Di Marco MD); Azienda Ospedaliera di Rilievo

Nazionale dei Colli, Cotugno

Hospital of Naples, Naples,

Italy (V Sangiovanni MD);

Gastroenterology Unit, Department of Clinical Medicine and Surgery,

Federico II University of Naples,

Naples, Italy (F Morisco MD); Internal Medicine, Department

of Medical Area, University of Udine, Udine, Italy (PToniutto MD);

Gastroenterology, Gemelli Foundation, Catholic University, Rome, Italy (A Tortora MD);

Gastroenterology Unit,

Hospital of Cosenza, Cosenza,

Italy (R De Marco MD); Liver Unit, Tor Vergata University, Rome, Italy

(Prof M Angelico MD); Division of Clinical and Molecular

Hepatology, University

Hospital of Messina, Messina,

Italy (I Cacciola PhD); Infectious

Diseases and Hepatology,

University Hospital of Parma,

Parma, Italy (G Elia MD);

Department of Clinical and Experimental Internal

Medicine, University of

Campania Luigi Vanvitelli,

Naples, Italy (A Federico PhD); Gastroenterology and

Endoscopy Unit, Foundation

Istituto di Ricovero e Cura a

Carattere Scientifico Ca' Granda

Ospedale Maggiore Policlinico,

Milan, Italy (S Massironi PhD);

Internal Medicine, S Sebastiano

General Hospital, Frascati
Research in context

\section{Evidence before this study}

We searched PubMed for full papers in any language published in peer-reviewed journals up to Feb 19, 2018, with the term "liver cirrhosis" subsequently filtered by "ascites", "human albumin", and "clinical trial". Among 122 publications retrieved, two reported the results of randomised clinical trials assessing the effects of long-term human albumin (HA) in patients with cirrhosis and ascites, whereas the others dealt with single or short-term $\mathrm{HA}$ administration to prevent or treat specific acute complications of the disease. In the first study, published in 1999, HA given at a dose of $25 \mathrm{~g}$ every week for 1 year and $25 \mathrm{~g}$ every 2 weeks for the next 2 years reduced the probability of developing ascites and hospital readmissions, with no effect on survival. An improved transplant-free survival was instead reported by the second study, published in 2006, by the same research group, in which patient follow-up was extended to a median of 84 months. However, the small sample size precluded a firm conclusion, so that current guidelines do not support such a treatment.

\section{Added value of this study}

The ANSWER study, an investigator-initiated multicentre randomised, parallel, open-label, pragmatic trial, is the first adequately powered study to assess the effects of long-term HA administration in patients with cirrhosis and uncomplicated ascites. Patients received either standard medical treatment (SMT, $\mathrm{n}=213$ ) or SMT plus HA ( $40 \mathrm{~g}$ twice weekly for 2 weeks, and then $40 \mathrm{~g}$ weekly; $\mathrm{n}=218$ ) for up to 18 months.

The all-cause 18-month mortality, the primary endpoint of the study, was evaluated as difference of events and analysis of survival time. The incidence rate of mortality was significantly lower in the SMT plus HA group than in the SMT group. Consistently, the overall 18-month survival was significantly higher in the SMT plus HA group than in the SMT group, resulting in a 38\% reduction in the mortality hazard ratio.

The SMT plus HA group also had significantly reduced incidence rates of paracentesis, refractory ascites, spontaneous bacterial peritonitis or other bacterial infections, episodes of renal dysfunction, hepatorenal syndrome type 1 , and hepatic encephalopathy grade 3-4, whereas the incidence rate of gastro-oesophageal variceal bleeding was similar in the two groups. Finally, SMT plus HA was associated with a better quality of life and fewer hospital admissions than the SMT group, contributing to a favourable incremental cost-effectiveness ratio. The expected result of long-term HA administration to patients with decompensated cirrhosis is conventionally believed to be a better management of ascites than SMT, as convincingly shown by the ANSWER study. However, and more importantly, it shows that long-term HA treatment also reduces the incidence of potentially fatal complications of end-stage liver disease, ultimately leading to an improved survival. Notably, this therapeutic approach appears to be cost-effective.

\section{Implications of all the available evidence}

The ANSWER study results are clinically relevant. Indeed, they might prompt a change in HA use from targeting specific complications to a more comprehensive approach aimed at slowing down the progression of decompensated cirrhosis by acting as a disease-modifying treatment. Long-term HA administration unavoidably requires the use of health-care services and a careful patient compliance. However, the clinical advantages achieved and the favourable results of cost-effectiveness analysis can justify the implementation of this management strategy. Future research should clarify whether some patient subgroups would benefit most from long-term HA treatment and whether different doses and timing schedules might be more effective than those used in the present study. abdominal fluid had been resolved by diuretics. ${ }^{9}$ Effective hypovolaemia due to peripheral arterial vasodilation is a hallmark of decompensated cirrhosis, leading to renal sodium and water retention that favours ascites formation. ${ }^{10}$ Therefore, a beneficial effect of HA might result from blood volume expansion, which would taper activated vasoconstrictor and sodium-retaining systems and improve renal perfusion. Sustained systemic inflammation and pro-oxidant state have been shown to contribute to circulatory and extrahepatic organ dysfunctions in advanced cirrhosis. ${ }^{11}$ HA exerts nononcotic properties, such as antioxidant and scavenging activities, binding and transport of exogenous and endogenous substances, and regulation of endothelial function and inflammatory or immune responses. ${ }^{12}$ Such properties would make HA potentially able to target several pathophysiological mechanisms underlying decompensated cirrhosis, providing another reason for the use of HA with the broader target of preventing complications, besides improving the management of ascites.

The effects of adding long-term HA administration to diuretic therapy in decompensated cirrhosis have been assessed by only one research group in two prospective controlled clinical trials. In the first trial, $\mathrm{HA}$ administration after hospital discharge at a dose of $25 \mathrm{~g}$ every week for 1 year and 25 g every 2 weeks for the following 2 years, reduced the probability of developing ascites and hospital readmissions, with no effect on survival..$^{13}$ The second trial extended patient follow-up to a median of 84 months, reporting improved transplant-free survival. ${ }^{14}$ However, the small sample size precluded a firm conclusion, and current guidelines do not support such a treatment. ${ }^{15,16}$

With this background and our large randomised clinical trial, we aimed to assess the effect of long-term HA administration on overall mortality, management of ascites, and incidence of complications in patients with decompensated cirrhosis. Health-care costs and quality of 
life were also assessed to estimate the cost-effectiveness of the intervention.

\section{Methods \\ Study design}

The human Albumin for the treatmeNt of aScites in patients With hEpatic ciRrhosis (ANSWER) study was an investigator-initiated multicentre randomised, parallel, open-label, pragmatic trial done in 33 academic and nonacademic Italian hospitals. Study protocol, amendments (appendix), and the informed consent process were first approved by the ethics committee at the coordinating centre (University of Bologna, S Orsola-Malpighi Hospital, Bologna, Italy) and then at each participating centre. This study fulfils the requirements of the CONSORT check list. ${ }^{17}$ We also self-reviewed the trial design and conduct using the Cochrane risk of bias tool (appendix). ${ }^{18}$

\section{Participants}

The target trial population comprised patients with cirrhosis and persistent uncomplicated ascites despite ongoing diuretic treatment, who were recruited at participating hospitals during hospital admissions or visits at outpatient clinics. Written informed consent was obtained from all patients and informants. The inclusion criteria were, diagnosis of liver cirrhosis with uncomplicated ascites; ongoing diuretic treatment with an antialdosteronic drug (at a dose $\geq 200 \mathrm{mg} /$ day and furosemide $\geq 25 \mathrm{mg}$ /day), stable for at least 4 days before enrolment; and oesophagogastroduodenoscopy done in the past 12 months, abdominal ultrasonography done in the past 30 days, and laboratory tests required by the protocol in the past 7 days. Participants younger than 18 years were excluded.

The main exclusion criteria aimed to avoid the enrolment of patients with refractory ascites, recent complications of cirrhosis, transjugular intrahepatic portosystemic shunt (TIPS), active hepatocellular carcinoma, liver transplantation, ongoing alcohol abuse, extrahepatic organ failure, and albumin use for the treatment of ascites in the month preceding enrolment. For a more detailed list of the exclusion criteria and the diagnostic criteria defining cirrhosis, ascites, and complications see the appendix.

\section{Randomisation and masking}

At the statistical data centre, eligible patients were randomly assigned (1:1) to receive either standard medical treatment (SMT) or SMT plus HA with a computergenerated and blinded assignment sequence with randomly permuted blocks of four, stratified according to the need for therapeutic paracentesis in the month preceding enrolment (yes or no) and natraemia ( $<135 \mathrm{mmol} / \mathrm{L}$ and $\geq 135 \mathrm{mmol} / \mathrm{L})$. A placebo controlled trial was not ethically justified because of the absence of safe alternatives to HA. Neither patients, nor investigators, nor statisticians were masked to treatment assignment. The entire statistical analysis was done once the database had been definitively frozen.

\section{Procedures}

SMT was aligned with the indications from the available clinical practice guidelines. ${ }^{15,16,19}$ According to the inclusion criteria, patients entering the study had to be treated with an anti-aldosteronic drug (at a dose $\geq 200 \mathrm{mg} /$ day) and furosemide ( $\geq 25 \mathrm{mg} /$ day). Details on patient SMT are reported in the appendix.

Patients enrolled in the SMT plus HA group received an intravenous infusion of $20 \% \mathrm{HA}$ in $50 \mathrm{~mL}$ vials in approximately 30-60 $\mathrm{min}$ at a dose of $40 \mathrm{~g}$ twice weekly for the initial 2 weeks, and $40 \mathrm{~g}$ weekly thereafter. The first dose was administered within $24 \mathrm{~h}$ after randomisation. According to protocol, HA was infused by nursing personnel in outpatient settings, such as hospital clinics, local health centres, or home-care services according to available facilities, whereas patients admitted to hospital received HA on the scheduled date during their hospital stay.

No concomitant medications were forbidden during the study. Additional information about causes and other treatments during the study are reported in the appendix.

After enrolment, patients were assessed monthly for up to 18 months or study interruption or death. At each visit, clinical, laboratory, and instrumental data (if needed) were collected by the attending physicians, including interviews

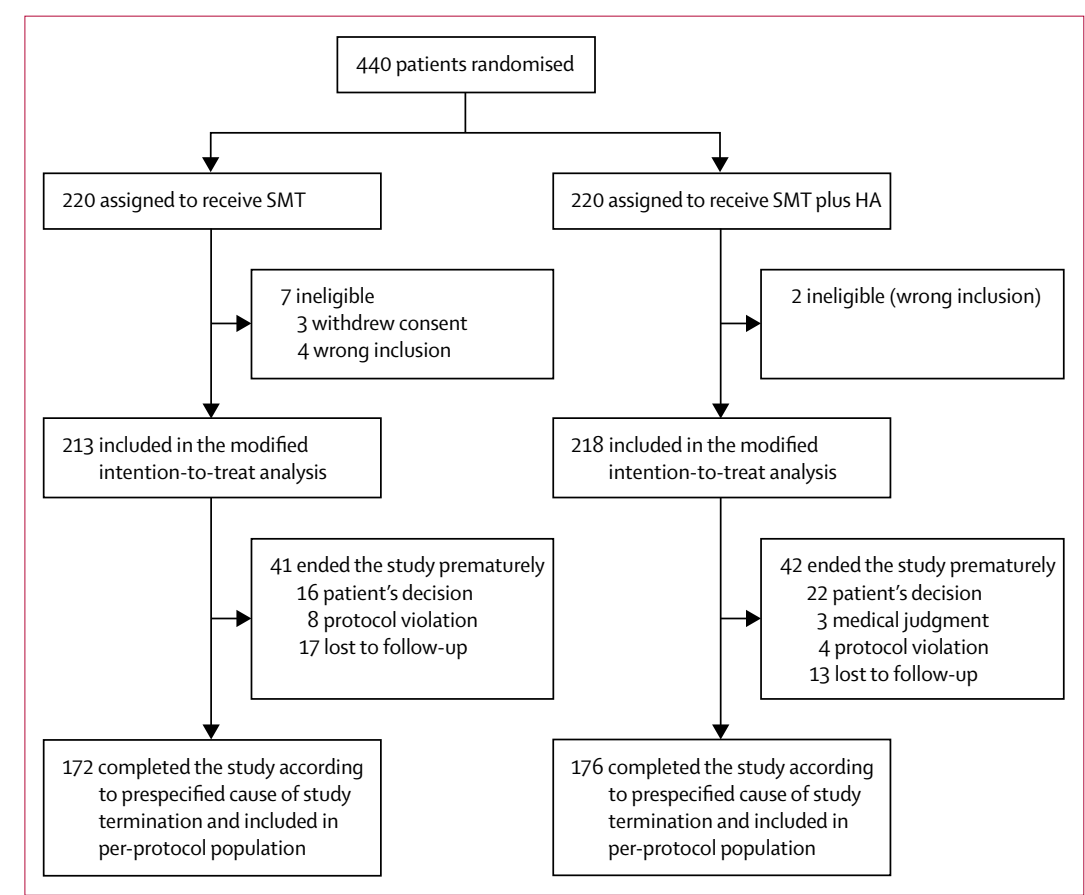

Figure 1: Trial profile

For the SMT group, wrong inclusions were one advanced hepatocellular carcinoma, one neoplastic ascites, and two refractory ascites. For the SMT plus HA group, wrong inclusions were one advanced hepatocellular carcinoma and one refractory ascites. $\mathrm{HA}=$ human albumin. SMT=standard medical treatment. 


\begin{tabular}{|c|c|c|}
\hline & SMT group $(n=213)$ & SMT plus HA group $(n=218)$ \\
\hline \multicolumn{3}{|l|}{ Demographic data } \\
\hline Age (years) & $61 \cdot 4(10 \cdot 9)$ & $61.0(11 \cdot 4)$ \\
\hline Male sex & $150(70 \%)$ & $146(67 \%)$ \\
\hline \multicolumn{3}{|l|}{ Cause of cirrhosis } \\
\hline Viral & $75(35 \%)$ & $72(33 \%)$ \\
\hline Alcohol & $69(32 \%)$ & $63(29 \%)$ \\
\hline NASH & $12(6 \%)$ & $8(4 \%)$ \\
\hline Viral and alcohol & $23(11 \%)$ & $36(17 \%)$ \\
\hline Alcohol and NASH & $11(5 \%)$ & $6(3 \%)$ \\
\hline Other & $23(11 \%)$ & $33(15 \%)$ \\
\hline \multicolumn{3}{|l|}{ Clinical features } \\
\hline Body-mass index $\left(\mathrm{kg} / \mathrm{m}^{2}\right)$ & $25 \cdot 4(23 \cdot 5-28 \cdot 2)$ & $25 \cdot 5(22 \cdot 8-28 \cdot 1)$ \\
\hline Systolic arterial pressure $(\mathrm{mm} \mathrm{Hg})$ & $110(110-120)$ & $115(110-125)$ \\
\hline Diastolic arterial pressure $(\mathrm{mm} \mathrm{Hg})$ & $70(60-80)$ & $70(60-75)$ \\
\hline Mean arterial pressure $(\mathrm{mm} \mathrm{Hg})$ & $83(80-90)$ & $83(68-91)$ \\
\hline Heart rate (beats per min) & $70(64-78)$ & $70(64-78)$ \\
\hline \multicolumn{3}{|l|}{ Ascites } \\
\hline Grade 2 & $173(81 \%)$ & $185(85 \%)$ \\
\hline Grade 3 & $40(19 \%)$ & $33(15 \%)$ \\
\hline Peripheral oedema & $102(48 \%)$ & $108(50 \%)$ \\
\hline Hepatic encephalopathy grade $1 / 2$ & $18(8 \%)$ & $21(10 \%)$ \\
\hline Hyponatraemia (<135 mmol/L) & $74(35 \%)$ & $75(34 \%)$ \\
\hline Portal thrombosis & $17(8 \%)$ & $18(8 \%)$ \\
\hline Inactive $\mathrm{HCC}$ & $2(1 \%)$ & $3(1 \%)$ \\
\hline \multicolumn{3}{|l|}{ Haematological and biochemical data } \\
\hline White blood cells $\left(10^{3}\right.$ cells per $\left.\mu \mathrm{L}\right)$ & $4 \cdot 79(3 \cdot 70-6 \cdot 40)$ & $5 \cdot 10(3 \cdot 90-6 \cdot 61)$ \\
\hline Haemoglobin (g/dL) & $11 \cdot 49(1.76)$ & $11.78(1.83)$ \\
\hline Platelets ( $10^{3}$ cells per $\mu \mathrm{L}$ ) & $90(60-128)$ & $95(69-133)$ \\
\hline Serum creatinine $(\mathrm{mg} / \mathrm{dL})$ & $0.98(0.80-1.16)$ & $0.91(0.79-1.10)$ \\
\hline Serum sodium (mmol/L) & $136(133-138)$ & $136(133-138)$ \\
\hline Serum potassium (mmol/L) & $4.34(0.54)$ & $4.37(0.56)$ \\
\hline Serum bilirubin ( $\mathrm{mg} / \mathrm{dL}$ ) & $1.88(1 \cdot 24-2 \cdot 80)$ & $1.83(1.09-3.20)$ \\
\hline Serum albumin (g/dL) & $3.10(0.49)$ & $3.09(0.55)$ \\
\hline International normalised ratio (INR) & $1.41(0.28)$ & $1.37(0.26)$ \\
\hline \multicolumn{3}{|l|}{ Prognostic scores } \\
\hline \multicolumn{3}{|l|}{ Child-Pugh class } \\
\hline Class A & $29(14 \%)$ & $35(16 \%)$ \\
\hline Class B & $141(66 \%)$ & $141(65 \%)$ \\
\hline Class C & $43(20 \%)$ & $42(19 \%)$ \\
\hline Child-Pugh score & $8(7-9)$ & $8(7-9)$ \\
\hline MELD score & $13(10-16)$ & $12(10-15)$ \\
\hline MELD-Na score & $16.43(4.57)$ & $15.91(4.90)$ \\
\hline \multicolumn{3}{|l|}{ Endoscopic findings } \\
\hline Oesophageal varices & $142(67 \%)$ & $144(66 \%)$ \\
\hline \multicolumn{3}{|l|}{ Oesophageal varices grade } \\
\hline $\mathrm{F} 1$ & $92(65 \%)$ & $84(58 \%)$ \\
\hline $\mathrm{F} 2$ & $44(31 \%)$ & $50(35 \%)$ \\
\hline F3 & $6(4 \%)$ & $10(7 \%)$ \\
\hline Gastric varices & $15(7 \%)$ & $20(9 \%)$ \\
\hline \multirow[t]{2}{*}{ Congestive gastropathy } & $151(71 \%)$ & $148(68 \%)$ \\
\hline & & (Table 1 continues on next page) \\
\hline
\end{tabular}

on dietary sodium and alcohol intake, which could be extended to relatives. Data were recorded on an electronic case report form accessible via the internet. The study was interrupted when patients underwent liver transplantation or TIPS insertion, needed three or more therapeutic paracenteses per month (the reason for this criterion is detailed in the appendix), or refused to continue their participation in the study, or because of medical judgment.

Quality of life was assessed with the visual-analogue scale (VAS) and the utility index from the EuroQol Group 5-Dimension Self-Report Questionnaire (EQ-5D). ${ }^{20,21}$ Contrary to what was planned in the study protocol, the Short Form 36 questionnaires were not used to assess quality of life because the number of questionnaires adequately completed was insufficient.

Adverse events were assessed at every visit from the time of first HA administration, and serious adverse events were monitored throughout the study. All adverse events were coded according to the Common Terminology Criteria for Adverse Events version 4.0. The severity and relation of adverse events to HA were reported according to the investigator's opinion.

\section{Outcomes}

The primary endpoint was 18-month mortality. The secondary endpoints were, (1) number of therapeutic paracenteses; (2) cumulative diuretic dosage; (3) hyponatraemia (serum sodium concentration $<130 \mathrm{mmol} / \mathrm{L}$ ) or hyperkalaemia (serum potassium concentration $\geq 5.5 \mathrm{mmol} / \mathrm{L}$ ) as potential diuretic-induced side-effects; (4) incidence of cirrhosis-related complications (refractory ascites, spontaneous bacterial peritonitis [SBP], other bacterial infections, renal impairment [serum creatinine concentration $>1.5 \mathrm{mg} / \mathrm{dL}]$, hepatorenal syndrome [HRS] type 1, hepatic encephalopathy grade 3 or 4 , and gastrointestinal bleeding related to portal hypertension); (5) need for three or more paracenteses per month; (6) quality of life; (7) number and duration of hospital admissions; and (8) treatment cost-effectiveness.

According to the study protocol, specific disease-related adverse events and serious adverse events were collected and documented as part of the clinical outcomes and hence were considered waived from expedited reporting to the Regulatory Authorities (appendix).

\section{Statistical analysis}

We assumed that the 18-month all-cause mortality would be $35 \%$ in the SMT group (according to the control group of a randomised clinical trial enrolling patients with clinical features similar to those of the patients in the present study ${ }^{22}$ and $20 \%$ in the SMT plus HA group. We assumed a drop rate of $4 \%$ per month and that patients were randomly assigned with 1:1 ratio, with a two-sided type 1 error rate of $5 \%$. Based on these assumptions, 210 patients per group were needed to ensure $90 \%$ power.

An interim analysis was planned in the protocol once half the patient sample size had completed the study 
(appendix). The final analysis was done on a modified intention-to-treat population. Descriptive statistics were generated for all variables. We used a $\chi^{2}$ test to compare categorical variables between SMT and SMT plus HA groups; we compared continuous variables using an unpaired $t$ test, Welch's test, or Mann-Whitney $U$ test, as appropriate. We calculated the median follow-up period according to the reverse Kaplan-Meier method. ${ }^{23}$

The primary efficacy endpoint, 18-month mortality, was defined as the number of deaths due to any cause during the follow-up or the time from randomisation to death from any cause. We assessed mortality by two methods: ratio of the number of observed deaths in person-18 months at risk of the event and analysis of survival time. We analysed all time-to-event distributions using the Kaplan-Meier's method (K-M), assessing differences with log-rank test. We calculated risk factors associated with survival by Cox proportional-hazards regression: hazard ratios (HR) and $95 \% \mathrm{CI}$ were estimated after confirmation of the proportional-hazards assumption. The univariate Cox proportional-hazards model was fit for all demographic and baseline variables considered relevant or clinically meaningful; we further analysed the factors showing statistical significance (at $\alpha$ level $0 \cdot 10$ ) as a predictor in univariate Cox proportionalhazard regression using a multivariable model with backward selection of variables based on the Akaike information criterion.

To assess secondary outcomes, we computed incidence rates and 95\% CI using the exact method on the basis of the Poisson distribution. Subsequently, to compare the incidence rates in the two groups, we calculated the incidence rate ratios and 95\% CI using the SMT group as the reference category.

We did a post-hoc competing risks analysis to test possible effects of liver transplantation and TIPS placement on the primary outcome. ${ }^{24}$ We did the same analysis, including all-cause deaths and TIPS placement or liver transplantation, to assess the treatment effect on the first occurrence of complications included in the secondary outcomes (appendix).

All reported $\mathrm{p}$ values are two-sided unless otherwise specified, and confidence intervals are at the 95\% level. Further details on the statistical methods are provided in the appendix.

We analysed cost-effectiveness by calculating qualityadjusted life-years (QALY) from the EQ-5D utility index and the incremental cost-effectiveness ratio (ICER). According to the National Institute for Health and Care Excellence (NICE), ${ }^{25}$ an ICER of $€ 35000$ per QALY was taken as the threshold to consider a treatment costeffective. We also did a non-parametric bootstrap analysis to test the robustness of the preliminary cost-effectiveness assessment (appendix). ${ }^{26}$

Data were managed with PL/SQL Developer, version 9.0.6.1665 (Allround automation, Enschede, Netherlands) and analyses were done by the non-profit Interuniversity

\begin{tabular}{|c|c|c|}
\hline & SMT group $(n=213)$ & SMT plus HA group $(n=218)$ \\
\hline \multicolumn{3}{|l|}{ (Continued from previous page) } \\
\hline \multicolumn{3}{|l|}{ Diuretic drugs } \\
\hline Antialdosteronic (mg per day) & $232.86(58 \cdot 28)$ & $244.84(66.68)$ \\
\hline Furosemide (mg per day) & $53 \cdot 76(30 \cdot 75)$ & $50 \cdot 46(26 \cdot 32)$ \\
\hline \multicolumn{3}{|l|}{ Concomitant medications } \\
\hline Beta blockers & $116(54 \%)$ & $107(49 \%)$ \\
\hline Antibiotic prophylaxis & $37(17 \%)$ & $46(21 \%)$ \\
\hline Quinolones & $7(3 \%)$ & $12(5 \%)$ \\
\hline Paromomycin & $0(0 \%)$ & $2(1 \%)$ \\
\hline Rifaximin & $30(14 \%)$ & $32(15 \%)$ \\
\hline Proton-pump inhibitors or $\mathrm{H}_{2}$ blockers & $166(78 \%)$ & $173(79 \%)$ \\
\hline \multicolumn{3}{|l|}{ Clinical history } \\
\hline \multicolumn{3}{|l|}{ Paracentesis } \\
\hline In past month & $55(26 \%)$ & $61(28 \%)$ \\
\hline In past 6 months & $103(48 \%)$ & $107(49 \%)$ \\
\hline \multicolumn{3}{|l|}{ Time from first ascitic decompensation } \\
\hline$\leq 12$ months & $152(71 \%)$ & $154(71 \%)$ \\
\hline$>12$ months & $61(29 \%)$ & $64(29 \%)$ \\
\hline Gastroesophageal variceal bleeding & $35(16 \%)$ & $27(12 \%)$ \\
\hline Hepatic encephalopathy & $57(27 \%)$ & $55(25 \%)$ \\
\hline SBP & $17(8 \%)$ & $18(8 \%)$ \\
\hline Non-SBP bacterial infections* & $30(14 \%)$ & $21(10 \%)$ \\
\hline Renal dysfunction $\dagger$ & $11(5 \%)$ & $15(7 \%)$ \\
\hline \multicolumn{3}{|l|}{ Number of decompensating eventsł } \\
\hline Only ascites & $106(49 \cdot 8 \%)$ & $125(57 \cdot 3 \%)$ \\
\hline Ascites and 1 decompensating event & $77(36 \cdot 2 \%)$ & $68(31 \cdot 2 \%)$ \\
\hline Ascites and 2 decompensating events & $27(12 \cdot 7 \%)$ & $22(10 \cdot 1 \%)$ \\
\hline Ascites and 3 decompensating events & $2(0 \cdot 9 \%)$ & $3(1 \cdot 4 \%)$ \\
\hline Ascites and 4 decompensating events & $1(0 \cdot 5 \%)$ & $0(0.0 \%)$ \\
\hline Ascites and $\geq 1$ decompensating event & $107(50 \cdot 2 \%)$ & $93(42 \cdot 7 \%)$ \\
\hline Patients in waiting list for LT & $17(8 \%)$ & $17(8 \%)$ \\
\hline Comorbidities & $132(62 \%)$ & $118(54 \%)$ \\
\hline Cardiovascular disease & $46(35 \%)$ & $46(39 \%)$ \\
\hline Pulmonary disease & $13(10 \%)$ & $18(15 \%)$ \\
\hline Gastro-intestinal disease & $23(17 \%)$ & $27(23 \%)$ \\
\hline Insulin-treated diabetes & $50(38 \%)$ & $35(30 \%)$ \\
\hline Genito-urinary disease & $17(13 \%)$ & $9(8 \%)$ \\
\hline \multicolumn{3}{|l|}{ Quality of life } \\
\hline EQ-5D utility index & $0.79(0.19)$ & $0.78(0.19)$ \\
\hline EQ-VAS score & $57 \cdot 2(18 \cdot 6)$ & $57 \cdot 2(19 \cdot 1)$ \\
\hline \multicolumn{3}{|c|}{$\begin{array}{l}\text { Data are } \mathrm{n}(\%) \text {, median (IQR) or mean }(\mathrm{SD}) . \mathrm{SMT}=\text { standard medical treatment. } \mathrm{HA}=\text { human albumin. } \\
\mathrm{NASH}=\text { non-alcoholic steatohepatitis. } \mathrm{HCC}=\text { hepatocellular carcinoma. } \mathrm{MELD}=\text { model for end stage liver disease. } \\
\text { MELD-Na=MELD score incorporating serum sodium concentration. } \mathrm{SBP}=\text { spontaneous bacterial peritonitis. } \mathrm{LT}=\text { liver } \\
\text { transplantation. EQ-5D=EuroQol Group 5-Dimension Self-Report Questionnaire. EQ-VAS=EuroQol visual analogue } \\
\text { scale. *Episodes requiring admission to hospital in the past } 3 \text { months. } † \text { Serum creatinine }>1.5 \mathrm{mg} / \mathrm{dL} \text { in the past } \\
3 \text { months. } \neq S B P \text {, non-SBP bacterial infections, gastrointestinal bleeding, hepatic encephalopathy. }\end{array}$} \\
\hline
\end{tabular}

Consortium for data collection, data processing, and statistical analysis (CINECA, Bologna, Italy) using R open-source statistical software, version 3.3.1.

The trial was monitored by an external contract research organisation (Yghea, Bologna, Italy) and overseen by a data safety and monitoring board of physicians not 


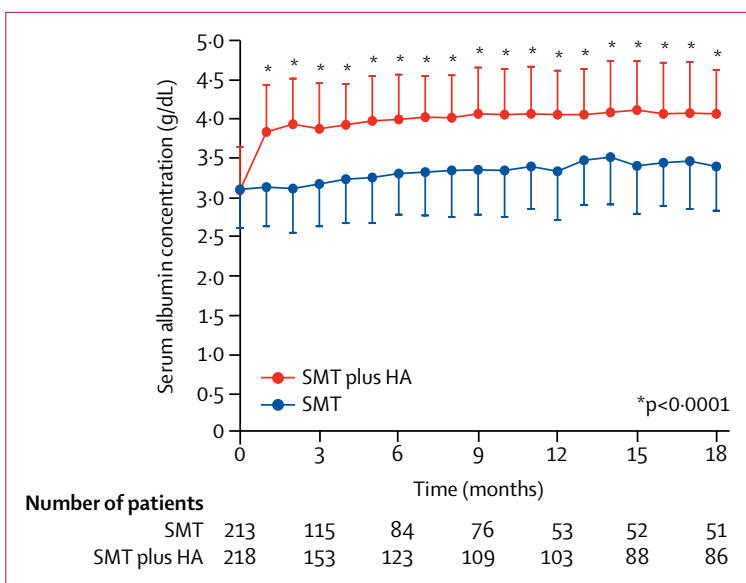

Figure 2: Serum albumin concentration throughout the study period Serum albumin concentration in patients receiving SMT or SMT plus HA. Dots are mean values and bars are SD. HA=human albumin. SMT=standard medical treatment.

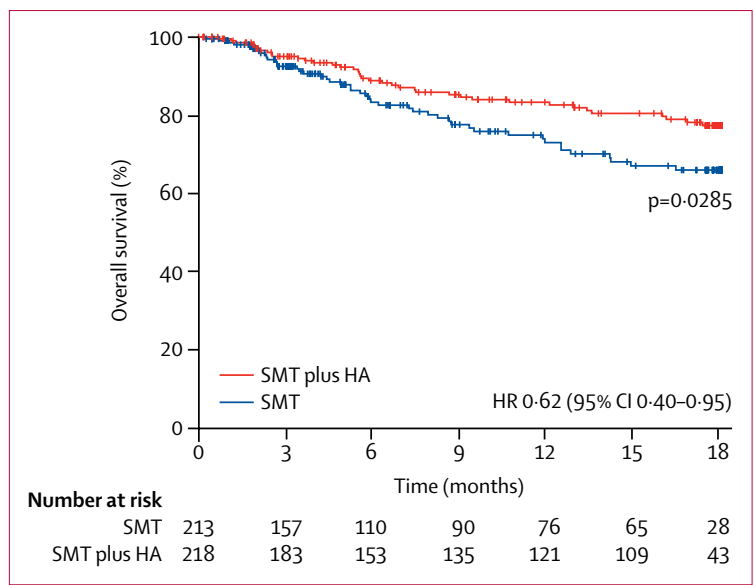

Figure 3: Overall survival

Kaplan-Meier estimates for the probability of overall survival in the modified intention-to-treat population of SMT and SMT plus HA groups. The $p$ value was calculated by the log-rank test. $\mathrm{HA}=$ human albumin. SMT=standard medical treatment.

involved in the study (appendix). Per-protocol HA was supplied by a company pool (Baxalta, CSL Behring, Biotest Italia, Grifols Italia, Kedrion Biopharma) according to Italian legislation governing non-profit trials.

The study was registered in the European Clinical Trial Database (EudraCT2008-000625-19) and ClinicalTrials. gov (NCT01288794).

\section{Role of the funding source}

The trial was funded by a competitive peer-reviewed grant from the Italian Medicine Agency. The funder had no role in study design, data collection, data analysis, data interpretation, or writing of the report. The corresponding author had full access to all the data in the study and had final responsibility for the decision to submit for publication.

\section{Results}

From April 2, 2011, to May 27, 2015, 440 patients were randomly assigned to receive either SMT $(n=220)$ or SMT plus HA $(n=220)$. Six patients, who were withdrawn from the study within 30 days from enrollment, were excluded because of wrong inclusion, and three patients, who did not receive any treatment, were excluded because of consent withdrawal. Therefore, the modified intention-totreat analysis included 213 patients in the SMT group and 218 in the SMT plus HA group (figure 1). Data on screened patients were incomplete in many participating centres, so comprehensive information about screening cannot be provided. However, screening was properly recorded at four highly recruiting centres where data were gathered (appendix). Baseline demographic, clinical, and laboratory features were well balanced between the two groups (table 1). 41 patients in the SMT group and 42 in the SMT plus HA group ended their follow-up prematurely. Thus, 172 patients in SMT group and 176 patients in SMT plus HA group completed the study according to the prespecified causes of study termination (per-protocol population). Although liver transplantation and TIPS placement were similar between the two groups, patients receiving SMT had a more frequent need for three or more paracenteses per month and had more deaths than those receiving SMT plus HA (figure 1) There were 46 deaths, 18 liver transplantations, 8 TIPS placements, and 42 needing three or more paracenteses per month in the SMT group and 38 deaths, 19 liver transplantations, 6 TIPS placements, and 18 needing three or more paracenteses per month in the SMT plus HA group. As a result, the follow-up was significantly shorter in the SMT group (median 11.5 months [IQR 3.3-17.8] vs 17.6 months [IQR $8 \cdot 0-18 \cdot 0] ; \mathrm{p}=0 \cdot 005)$. Details on the causes of premature study termination and deaths and protocol adherence are reported in the appendix.

In the SMT plus HA group, long-term HA administration was associated with a significant increase in mean serum albumin concentration from a baseline concentration of $3 \cdot 1 \mathrm{~g} / \mathrm{dL}$ to about $4 \mathrm{~g} / \mathrm{dL}$ within $1-2$ months $(\mathrm{p}<0 \cdot 0001)$, whereas no change occurred in the SMT group. Thereafter, serum albumin concentration remained stable and significantly higher in the SMT plus HA group than in the SMT group, with a gap fluctuating between 0.6 and $0.8 \mathrm{~g} / \mathrm{dL}(\mathrm{p}<0 \cdot 0001$; figure 2$)$.

The 18-month all-cause mortality rate was significantly lower in the SMT plus HA group $(0 \cdot 27$ deaths per person-18 months [95\% CI 0.19-0 37]) than in the SMT group $(0 \cdot 44$ [95\% CI $0 \cdot 32-0 \cdot 80])$, leading to an incidence rate ratio of 0.61 (95\% CI $0.39-0.96 ; \quad \mathrm{p}=0.027)$. Consistently, patients receiving HA had a significantly higher 18-month probability of overall survival than did those treated with SMT alone (K-M 77\% SMT plus HA vs $66 \%$ SMT; $\mathrm{p}=0 \cdot 0285$; figure 3 ), corresponding to a $38 \%$ reduction in the mortality $\operatorname{HR}(0.62$ [95\% CI $0 \cdot 40-0 \cdot 95])$. The beneficial effect of HA administration on 18-month all-cause mortality (incidence rate $0 \cdot 30$ [95\% CI 0 21-0 - 41] 
in SMT plus HA group; incidence rate $0 \cdot 48[0 \cdot 35-0 \cdot 64]$ in SMT group; incidence rate ratio 0.62 [0.39-0.98]; $\mathrm{p}=0.036)$ and overall survival (HR 0.63 [0.41-0.98]; K-M 75\% SMT plus HA vs 64\% SMT; $\mathrm{p}=0 \cdot 037$ ) was also observed in the per-protocol population. Univariate analysis (appendix) and multivariable Cox proportional hazard model (appendix) showed that age, viral cause of cirrhosis, Child-Pugh, and Model for End-Stage Liver Disease score incorporating serum sodium concentration were independent predictors of all-cause mortality, whereas receiving SMT plus HA was the sole protective factor. Finally, multivariable Cox regression analysis estimated that seven patients (95\% CI 5-19) were to be treated to avoid death at 18 months. Notably, the mixed effect Cox model showed a non-significant centre effect on primary outcome.

The competing risks analysis, including TIPS placement or liver transplantation as competing events with mortality, showed that the cumulative incidence of death was significantly reduced in the SMT plus HA group (figure 4A). Multivariable competing risks analysis, including the same set of factors selected in the multivariable Cox proportional hazard model, supported the independent protective role of HA administration against all-cause mortality (figure 4B). The analysis done, including liver-related mortality, non-liver-related mortality, and TIPS placement or liver transplantation, showed that the effect of HA administration was specific for liver-related deaths (figure 5).

Ascites control was substantially eased by long-term HA administration. Indeed, 71 patients had at least one paracentesis in the SMT plus HA group and 116 in the SMT group. The probability of remaining free of paracentesis throughout the study was almost twice in the SMT plus HA group (HR 0.48 [95\% CI 0.35-0.54]; K-M 62\% SMT plus HA vs 34\% SMT; p $<0 \cdot 0001)$ (figure 6A). Furthermore, the incidence rate of paracentesis in the SMT group was 3.50 cases per person-year (95\% CI $3 \cdot 21-3 \cdot 80$ ), which fell by $54 \%$ in patients receiving SMT plus HA (incidence rate 1.55 [95\% CI 1.39-1.73]; incidence rate ratio 0.46 [95\% CI $0 \cdot 40-0.53] ; \mathrm{p}<0.0001)$. The mean volume tapped per each paracentesis did not differ between the two groups (SMT, 5.5 L [SD 2.1]; SMT plus HA, 5.7 L [SD 2.1]; $\mathrm{p}=0 \cdot 51)$. Finally, the cumulative diuretic doses were similar in the two groups (appendix).

The cumulative incidence of refractory ascites was 0.25 in the SMT plus HA group and 0.48 in the SMT group ( $\mathrm{p}<0.0001)$, with a $57 \%$ risk reduction (HR 0.43 [95\% CI 0.29-0 62]; $\mathrm{p}<0 \cdot 0001$ ) (figure 6B).

Consistently, the cumulative incidence of the endpoint need for three or more paracenteses per month was lower in the SMT plus HA group than in the SMT group (K-M 12\% vs 29\%; p<0.0001), with a $67 \%$ risk reduction (HR 0.33 [95\% CI 0.19-0.58]; p<0.0001). Notably, the mean volume of ascites tapped for each of the last three paracenteses did not differ between the two groups

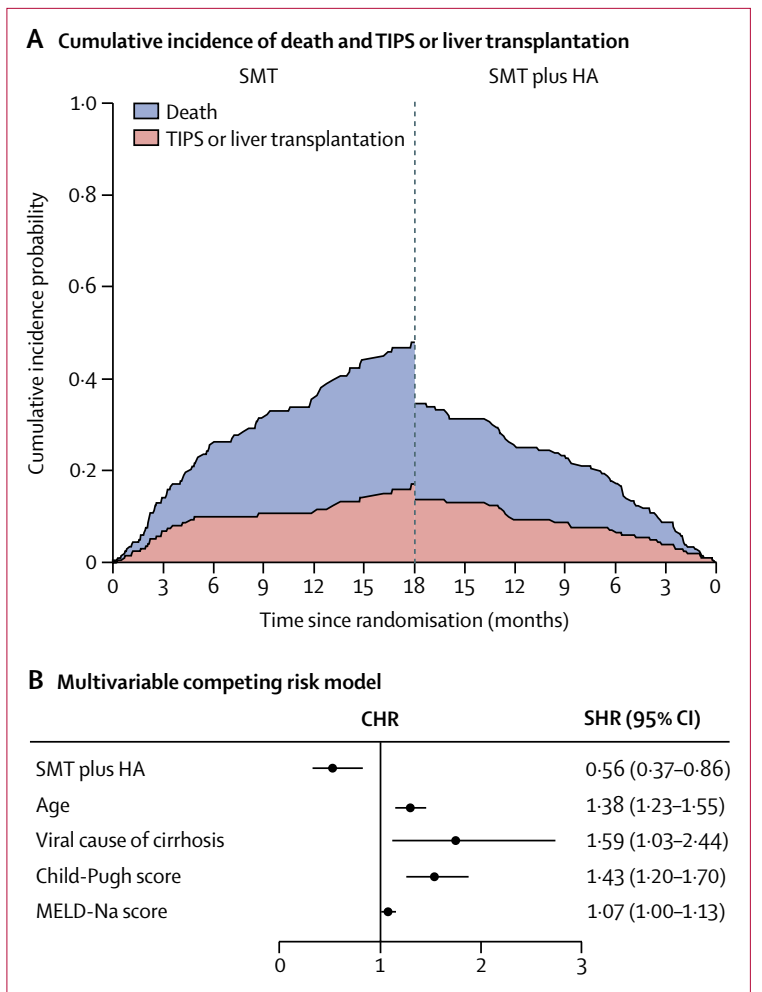

Figure 4: Competing risks analyses for 18-month all-cause mortality (A) Cumulative incidence of death and TIPS placement or liver transplantation in SMT and SMT plus HA groups. (B) CHR with bars indicating $95 \% \mathrm{Cl}$ and SHR with $95 \% \mathrm{Cl}$, according to the competing risk multivariable model in which TIPS placement or liver transplantation are competing events. Age (5-year increase), viral cause of cirrhosis (yes or no), Child-Pugh score (1-point increase), and MELD-Na score (1-point increase) were independent predictors of all-cause mortality, whereas SMT plus HA (yes or no) was the sole variable associated with survival. TIPS=transjugular intrahepatic portosystemic shunt. SMT=standard medical treatment. $\mathrm{HA}=$ human albumin. $\mathrm{CHR}=$ cause-specific hazard ratio. $\mathrm{SHR}=$ subdistribution hazard ratio. MELD-Na=Model for End-Stage Liver Disease score incorporating serum sodium concentration.

(SMT, 6·22 L [SD 1·58]; SMT plus HA, 6 ·1 L [SD 1·50]; $\mathrm{p}=0 \cdot 27)$.

Besides the benefit on ascites management, long-term HA administration appeared to protect patients against many other complications of cirrhosis. The incidence rate ratio of SBP, non-SBP bacterial infections, renal dysfunction, HRS type 1 , hepatic encephalopathy grade 3 or 4 , and potential diuretic-induced side-effects, such as hyponatraemia and hyperkalaemia, documented that the cumulative incidence of these complications was significantly reduced by 30 to $67.5 \%$ in patients receiving SMT plus HA (figure 7). Bleeding from gastro-oesophageal varices did not differ between the two groups; however, a non-significant trend to a higher incidence of other portal-hypertensive bleeds (congestive gastropathy or haemorrhoids) was seen in the SMT plus HA group (figure 7).

The cumulative incidence of major complications was also assessed by a competing risks analysis in which all-cause mortality and TIPS placement or liver 


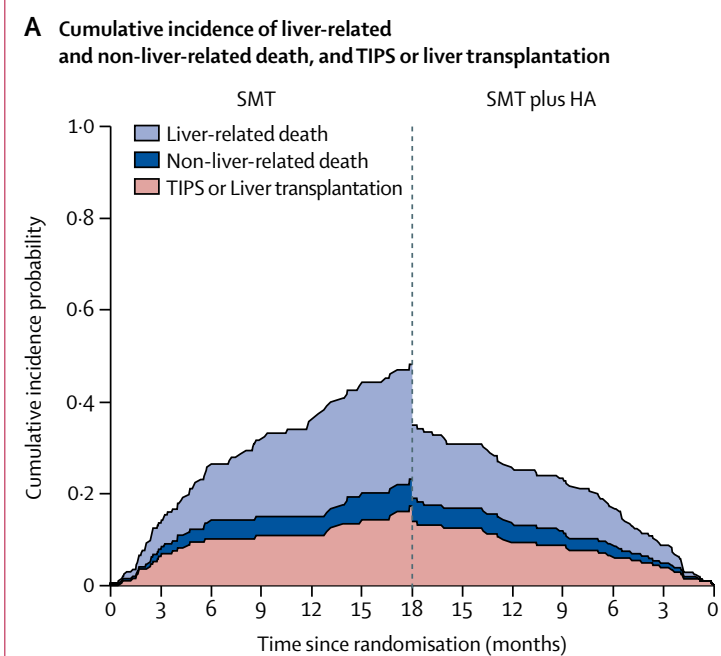

B Multivariable competing risk mode

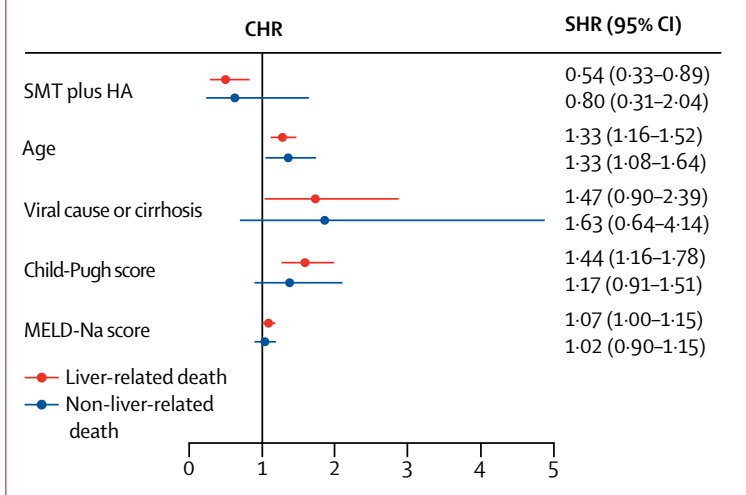

Figure 5: Competing risks analysis for 18-month liver-related and non-liver-related mortality

(A) Cumulative incidence of liver-related deaths, non-liver-related deaths, and TIPS placement or liver transplantation in SMT and SMT plus HA groups. (B) CHR with bars indicating $95 \% \mathrm{Cl}$ and SHR with $95 \% \mathrm{Cl}$, according to the competing risk multivariable model in which liver-related deaths, non-liver-related deaths, and TIPS placement or liver transplantation are competing events. Age (5-year increase), viral cause of cirrhosis (yes or no), Child-Pugh score (1-point increase), and MELD-Na score (1-point increase) were independent predictors of

liver-related mortality, whereas SMT plus HA (yes or no) was the sole variable associated with survival. Instead, age was the sole independent predictor of non-liver-related mortality. TIPS=transjugular intrahepatic portosystemic shunt SMT=standard medical treatment. HA=human albumin. $\mathrm{CHR}=$ cause-specific hazard ratio. SHR=subdistribution hazard ratio. MELD-Na=Model for End-Stage Liver Disease score incorporating serum sodium concentration.

transplantation were the competing events. This analysis showed a beneficial effect conferred by HA administration on refractory ascites and the first occurrence of HRS type 1, SBP, and hepatic encephalopathy grade 3 or 4 , whereas no effect was seen on the first occurrence of non-SBP bacterial infections and bleeding from gastro-oesophageal varices (appendix).

Quality of life throughout the follow-up, as assessed by mean values of EQ-5D utility index and VAS, remained roughly steady in the SMT plus HA group, whereas it

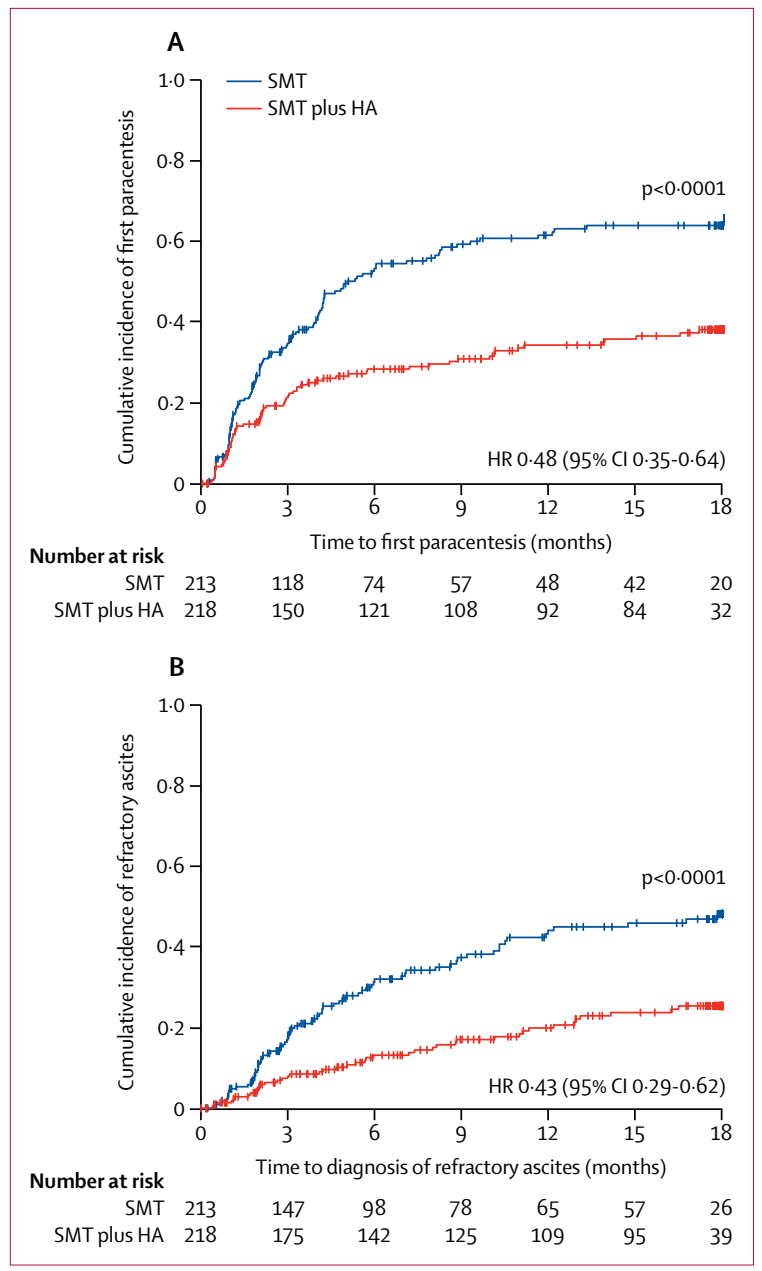

Figure 6: Management of ascites

Kaplan-Meier (cumulative event curves) estimates for (A) the probability of first paracentesis and (B) the probability of developing refractory ascites in patients randomly assigned to receive SMT or SMT plus HA. HA=human albumin. $\mathrm{HR}=$ hazard ratio. SMT=standard medical treatment. HR=hazard ratio.

declined to a significant extent from 3 to 12 months in the SMT group (appendix). The number of hospital admissions and days spent in hospital per patient in a year were significantly reduced in the SMT plus HA group by $35 \%$ and $45 \%$ with respect to the SMT group (table 2). Liver-related and non-liver-related causes of hospital admissions are reported in the appendix. For the cost-effectiveness analysis, direct health-care costs were based on the tariffs of the Italian National Health Service and included hospital admissions, procedures for perprotocol HA infusion, amount of HA administered for any reason, and paracentesis. The extra cost related to the per-protocol HA administration in the SMT plus HA group was largely counterbalanced by savings derived from fewer hospital admissions and paracenteses, and less HA use for established indications, such as prevention of paracentesis-induced circulatory dysfunction and SBP-induced renal dysfunction, and 


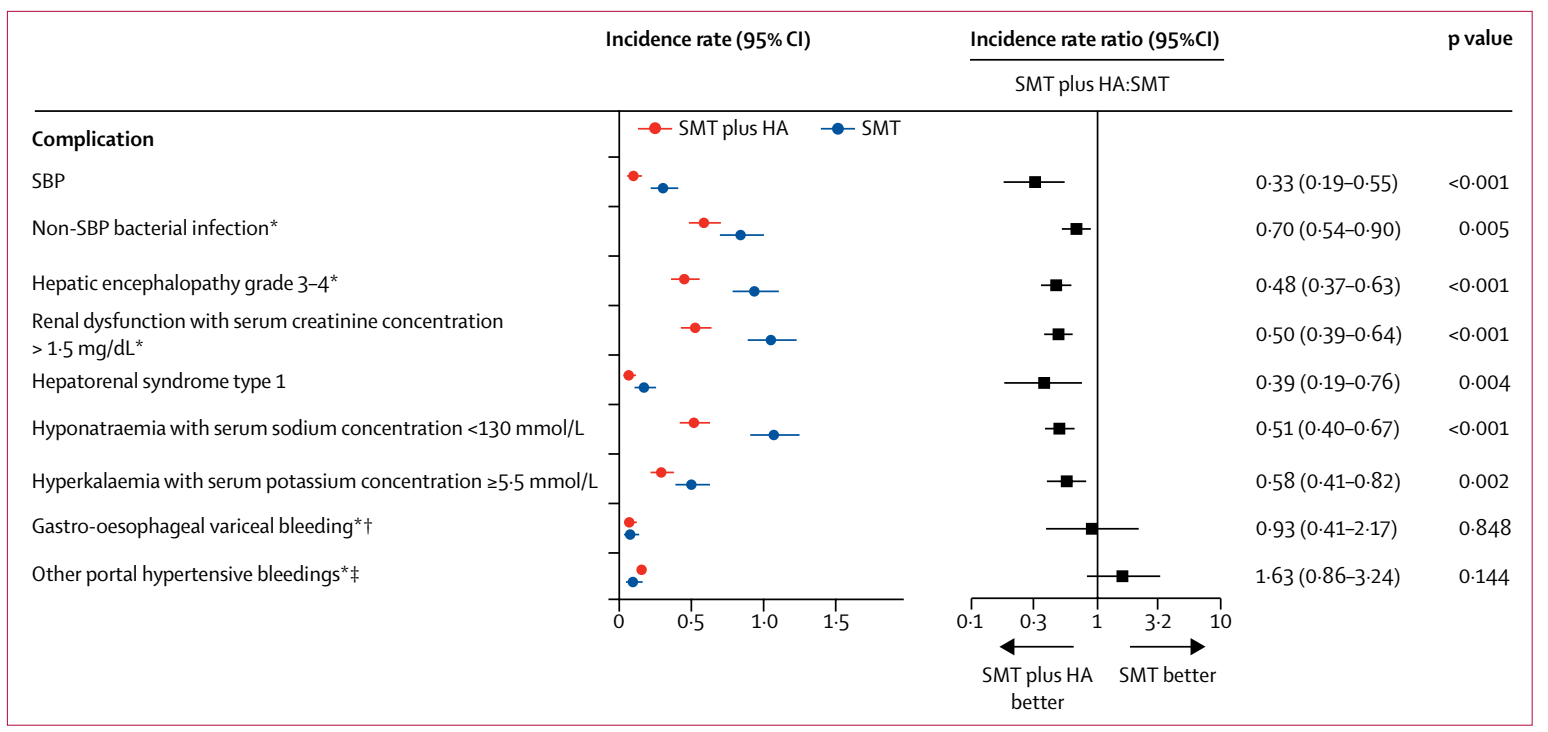

Figure 7: Complications of cirrhosis

Incidence rate (left), expressed as number of events per person per year (bars report 95\% Cl), of cirrhosis complications in patients randomly assigned to SMT plus HA or SMT. The incidence rate ratio (right) is the ratio of the incidence rate of each complication in the SMT plus HA group to the incidence rate of the same complication in the SMT group (SMT plus HA:SMT). The incidence rate ratio with its $95 \% \mathrm{Cl}<1$ indicates a significant reduction in the SMT plus $\mathrm{HA}$ group. $\mathrm{HA}=$ human albumin. $\mathrm{SBP}=$ spontaneous bacterial peritonitis. SMT=standard medical treatment. ${ }^{*}$ Complications not included as prespecified secondary endpoints in the original protocol of the study. †Due to rupture of oesophageal or gastric varices. $¥ D$ Due to congestive gastropathy or rectal varices.

treatment of HRS type 1 . As a result, an incremental cost of $€ 2488 /$ year was seen in the SMT plus HA group. Because the incremental health benefit achieved in the SMT plus HA group, estimated by the gain of QALY, was $0 \cdot 117$ QALY per year, the ICER was €21265 per QALY (appendix). The bootstrap analysis produced even more favourable results. In $56 \%$ of the 10000 simulations, long-term HA administration resulted in cost-saving compared with SMT alone. Moreover, $92.5 \%$ of all simulations presented an ICER of less than $€ 35000$ per QALY (appendix).

Adverse events temporally related to HA infusion included two mild allergic reactions consisting of erythema and pruritus, one episode of dizziness with transient arterial hypotension, and two severe sepsis cases. In the two cases of severe sepsis, bacteriological analysis of HA batches did not reveal contamination, and concomitant pneumonia was diagnosed in one case. All patients fully recovered (appendix). None of the unanticipated grade 3 and 4 adverse events considered unrelated to cirrhosis exceeded the 5\% frequency threshold within SMT and SMT plus HA groups (table 3). The non-liver-related serious adverse events requiring admission to hospital are listed in the appendix.

\section{Discussion}

The present randomised, multicentre, pragmatic trial is the first prospective study providing robust evidence for a survival advantage conferred by the addition of longterm HA administration to the standard-of-care in a large cohort of patients with cirrhosis and uncomplicated ascites. This statement is supported by the findings

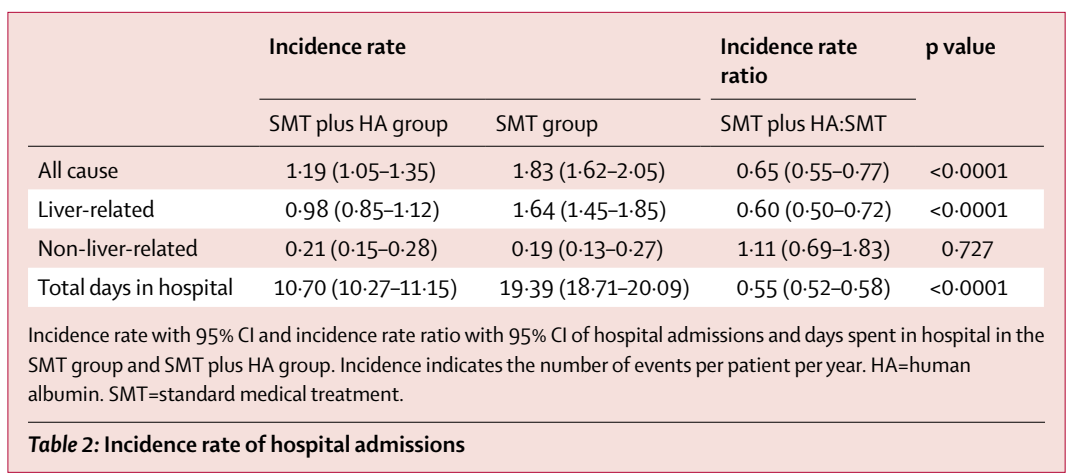

that HA administration significantly reduced overall mortality rate, thus improving 18-month survival. The benefit of HA administration was supported when TIPS placement and liver transplantation were considered competing risk events. Moreover, long-term HA treatment was the sole independent protective factor against mortality at competing risks multivariable analysis and this advantage was specific to liver-related deaths. The fact that only seven patients had to be treated to save a life heightens the clinical relevance of these results.

An improved survival of patients with cirrhosis and first-onset ascites receiving long-term HA was reported after a median follow-up of 84 months by a single-centre randomised trial ${ }^{14}$ more than a decade ago. However, its small patient sample size precluded the adoption of this treatment by current clinical practice guidelines. ${ }^{15,16} \mathrm{We}$ believe that the much larger, adequately powered patient sample size, and the more stringent methods adopted 


\begin{tabular}{|c|c|c|c|c|c|c|c|}
\hline & \multicolumn{2}{|l|}{ SMT $(n=213)$} & \multicolumn{2}{|c|}{ SMT plus HA (n=218) } & \multicolumn{2}{|l|}{ Total $(n=431)$} & \multirow[t]{2}{*}{$\mathrm{p}$ value } \\
\hline & $\begin{array}{l}\text { Patients with } \\
\text { event (\%) }\end{array}$ & Total events & $\begin{array}{l}\text { Patients with } \\
\text { event }(\%)\end{array}$ & Total events & $\begin{array}{l}\text { Patients with } \\
\text { event (\%) }\end{array}$ & Total events & \\
\hline Any event & $46(22 \%)$ & 53 & $49(22 \%)$ & 60 & $95(22 \%)$ & 113 & 0.825 \\
\hline $\begin{array}{l}\text { Injury, poisoning, and procedural } \\
\text { complications }\end{array}$ & $9(4 \%)$ & 10 & $10(5 \%)$ & 14 & $19(4 \%)$ & 24 & 0.855 \\
\hline Gastrointestinal disorders & $10(5 \%)$ & 13 & $9(4 \%)$ & 10 & $19(4 \%)$ & 23 & 0.775 \\
\hline Nervous system disorders & $5(2 \%)$ & 5 & $6(3 \%)$ & 7 & $11(3 \%)$ & 12 & 0.790 \\
\hline Cardiac disorders & $8(4 \%)$ & 8 & $3(1 \%)$ & 4 & $11(3 \%)$ & 12 & $0 \cdot 117$ \\
\hline $\begin{array}{l}\text { Respiratory, thoracic, and mediastinal } \\
\text { disorders }\end{array}$ & $2(1 \%)$ & 2 & $6(3 \%)$ & 6 & $8(2 \%)$ & 8 & 0.163 \\
\hline Blood and lymphatic system disorders & $2(1 \%)$ & 3 & $4(2 \%)$ & 5 & $6(1 \%)$ & 8 & 0.427 \\
\hline Metabolism and nutrition disorders & $3(1 \%)$ & 3 & $3(1 \%)$ & 4 & $6(1 \%)$ & 7 & 0.977 \\
\hline $\begin{array}{l}\text { Neoplasms benign, malignant, and } \\
\text { unspecified (including cysts and polyps) }\end{array}$ & $3(1 \%)$ & 4 & $1(<1 \%)$ & 2 & $4(1 \%)$ & 6 & 0.304 \\
\hline Renal and urinary disorders & $1(<1 \%)$ & 2 & $1(<1 \%)$ & 1 & $2(<1 \%)$ & 3 & 0.987 \\
\hline Psychiatric disorders & $0(0 \%)$ & 0 & $1(<1 \%)$ & 2 & $1(<1 \%)$ & 2 & 0.322 \\
\hline Eye disorders & $0(0 \%)$ & 0 & $2(1 \%)$ & 2 & $2(<1 \%)$ & 2 & $0 \cdot 161$ \\
\hline $\begin{array}{l}\text { Musculoskeletal and connective tissue } \\
\text { disorders }\end{array}$ & $1(<1 \%)$ & 1 & $1(<1 \%)$ & 1 & $2(<1 \%)$ & 2 & 0.987 \\
\hline Surgical and medical procedures & $1(<1 \%)$ & 1 & $1(<1 \%)$ & 1 & $2(<1 \%)$ & 2 & 0.987 \\
\hline Reproductive system and breast disorders & $0(0 \%)$ & 0 & $1(<1 \%)$ & 1 & $1(<1 \%)$ & 1 & 0.322 \\
\hline Vascular disorders & $1(<1 \%)$ & 1 & $0(0 \%)$ & 0 & $1(<1 \%)$ & 1 & $0 \cdot 311$ \\
\hline
\end{tabular}

in the present trial overcome the main limitations of that study.

Our study also provided other major findings. HA administration eased the management of ascites by significantly reducing the need for therapeutic paracentesis. The decision to perform paracentesis might be largely influenced by the subjective judgment of attending physicians. Therefore, the fact that the amount of ascitic fluid withdrawn per paracentesis was the same in the two groups of our study is notable, suggesting a fairly standard behaviour among investigators. The favourable effect of HA administration on ascites management is also testified by the significant reductions in the incidence of refractory ascites and diuretic-related side-effects, such as renal dysfunction, hyponatraemia, and hyperkalaemia, in the SMT plus HA group. Even more importantly, the incidence of ominous complications of cirrhosis, such as SBP and non-SBP-related bacterial infections, HRS type 1, and severe hepatic encephalopathy, was also strikingly reduced. These results were reinforced by the competing risks analyses showing that $\mathrm{HA}$ administration was associated with a delay in the occurrence of the first episodes of these complications apart from non-SBP bacterial infections. These effects probably represented the basis for the improved survival of patients receiving longterm HA. The diagnosis of hepatic encephalopathy and non-SBP bacterial infections must be recognised as difficult and potentially influenced by subjective interpretation. To temper these potential sources of bias, we only recorded the episodes of hepatic encephalopathy grade 3 and 4, with which clinical evidence is more straightforward, and used prespecified diagnostic criteria for non-SBP bacterial infections that follow those used in many studies specifically dealing with this issue..$^{27,28}$

Bleeding from oesophageal varices, likely to be caused by blood volume expansion after the prolonged administration of huge amounts of HA (up to $100 \mathrm{~g} /$ day), was reported many years ago in anecdotal series..$^{929,30}$ Thus, another important finding is that gastro-oesophageal variceal bleeding and related deaths were not increased by the HA dose given in the present study. We observed a non-significantly higher incidence of other portalhypertensive bleeds-ie, from portal gastropathy and haemorrhoids-with a higher hospital admission rate, without any increase in the related mortality. Whether or not this result is favoured by the mild anti-platelet aggregation effect of $\mathrm{HA}^{31}$ remains to be determined.

The nature of this study cannot unveil the mechanisms underlying HA benefit. However, HA could have mitigated effective hypovolaemia, which is a major pathogenic factor for ascites formation inducing renal sodium retention and endangering renal perfusion. ${ }^{10}$ This mitigation would explain a better control of ascites and related complications, such as renal impairment, electrolyte disturbances, HRS type 1, and SBP. Moreover, HA non-oncotic properties could have antagonised mechanisms, leading to the systemic inflammation and immune dysfunction characterising decompensated cirrhosis. ${ }^{11,12}$ This result would account for the significant reduced incidence of complications not closely linked to 
haemodynamic alterations, such as hepatic encephalopathy and non-SBP bacterial infections. Whether a serum albumin concentration threshold needs to be reached to achieve therapeutic effects from HA supplementation warrants further studies. A significant and sustained increase in serum albumin concentration was seen in the SMT plus HA group with respect to SMT. This difference was reached within 1-2 months, when the benefits of HA started to be manifest.

HA administration also influenced patients' quality of life. Under baseline conditions, the EQ-5D values were similar in the two groups, and close to a report in Italian patients with cirrhosis. ${ }^{32}$ During the follow-up, a lower decay of both utility index and VAS score was seen in the SMT plus HA than the SMT group. The resulting differences reached significance from the 3rd to the 12th month, a relevant result in such severely ill patients.

Long-term HA administration is deemed to be an expensive treatment, and an attempt to determine the costeffectiveness of this intervention is warranted. Hospital admissions represent a major health-care cost in patients with decompensated cirrhosis. ${ }^{6}$ Thus, an important result of our study is that HA treatment was associated with a significant reduction in the number of hospital admissions and total days spent in the hospital. These findings, combined with fewer paracenteses, lower expenditure to treat complications, and reduced HA use for evidencebased indications, are likely to balance the costs of longterm HA administration. In Italy, cost drivers are based on National Health Service reimbursement rates and are much lower than in other high-income countries. Despite this, the ICER:QALY ratio calculated in this study was below the threshold adopted by NICE to consider a treatment cost-effective. ${ }^{25}$ Furthermore, the bootstrap analysis further supported this finding, providing even more favourable results.

Long-term HA administration was generally very well tolerated as only three mild allergic reactions and two severe side-effects-represented by sepsis-occurred, leading to treatment interruption in three cases. HA batches analysis in the cases of sepsis did not reveal contamination, suggesting that these side-effects were due to venepuncture. Moreover, a concomitant previously unrecognised pneumonia in one patient casts doubts on the role of HA administration in that case.

The main limitation of this study is that it is an openlabel trial. Weekly HA administration led patients to be seen, usually by nurses, more frequently than those enrolled in the SMT group. Thus, impending complications could have been treated more promptly. However, closer patient surveillance might have disclosed a higher number of events, thereby increasing the incidence of complications in the SMT plus HA group. In any case, such a difference reflects what would happen in real-world clinical practice, a feature of pragmatic trials. ${ }^{33}$ Although pragmatic trials might have a reduced internal validity because of the absence of blinding, they are likely to have a better external validity provided they are founded on large sample-size, prolonged follow-up, and hard primary endpoints. All these features were observed in the present study, involving centres with diverse clinical settings. Notably, no significant centre effect was seen on primary outcome. Furthermore, besides the ethical concern related to the weekly patient attendance to hospital services to receive placebo infusion, the choice of the fluid to be used in patients with decompensated cirrhosis can be problematic. Indeed, any potential alternative presents shortcomings: sodium-containing solutions would enhance sodium overload, glucose solution is unsuitable in diabetics and might favour hyponatraemia, and synthetic colloids are potentially associated with side-effects such as anaphylaxis, coagulopathy, or renal failure. ${ }^{3436}$ Lastly, outcome assessors and analysts were also not blinded. However, the outcome analysts belong to an independent non-profit consortium (CINECA, Bologna, Italy) and did the entire statistical assessment once the database had been definitively frozen. This precaution would at least temper the risk of bias related to this type of unmasking.

A second limitation stems from the ethical need to interrupt the study when patients needed three or more paracenteses per month (appendix). This shortened the follow-up duration especially in the SMT group. However, censoring patients in a very advanced disease stage, when event incidence is likely to be higher, might have lessened rather than magnified the differences between the two groups. Finally, weekly intravenous infusions require frequent use of health-care services. However, the entire procedure can be done in less than $1 \mathrm{~h}$ in an outpatient setting or even at home, if domiciliary care is available. Even though the weekly schedule entails careful patient compliance, this study shows that most patients were strongly committed to follow a demanding treatment if they perceived a health benefit from therapy. We believe that the clinical advantages, including the lives saved and fewer admissions to hospital, justify the HA care management.

Another limitation regards the cost-effectiveness analysis as non-health care or indirect costs were not accounted for in our study. However, our cost analysis followed a thirdparty payer perspective that provides reimbursement of the Diagnosis Related Group and ambulatory tariffs, which include the cost of personnel but not indirect costs.

In conclusion, long-term weekly HA administration in patients with decompensated cirrhosis acts as a costeffective disease-modifying treatment since it not only provides a better control of ascites but also reduces the incidence of complications and related hospital admissions, and improves survival and quality of life. The identification of patient subgroups who would benefit most from long-term HA administration merits additional investigation. These results might prompt a change in HA use in decompensated cirrhosis from targeting specific complications to a more comprehensive approach aimed at slowing down the progression of the disease. 


\section{Contributors}

The original concept of the ANSWER trial was developed by MBe and PC. The study was designed and planned by PC, OR, PA, and MBe. The chief investigators were $\mathrm{MBe}$ and $\mathrm{PC}$. Acquisition of the data was done by PC, OR, PA, CA, SNe, FGF, FL, AAi, SB, GS-B, SF, RGR, RC, VDM, VS, FM, PT, AT, RDM, MA, IC, GE, AF, SM, RG, AG, GB, MRe, SNa, SP, CE, LP, FMC, LC, LS, CP, MC, AAn, FF, MT, and GZ. Statistical analysis was done by AR and MBa. Cost-effectiveness analysis was done by MRu. Data analysis and interpretation was done by PC, MBe, MT, $\mathrm{GZ}$, and MBa. The manuscript was drafted by PC and MBe. All authors critically reviewed the manuscript and approved the final draft for submission. The guarantor of the manuscript is MBe.

\section{ANSWER study investigators}

Marco Domenicali, Ferdinando A Giannone (Department of Medical and Surgical Sciences, University of Bologna); Manuela Merli, Stefania Gioia (Department of Clinical Medicine, Sapienza University of Rome); Silvano Fasolato, Antonietta Sticca (Unit of Internal Medicine and Hepatology, Department of Medicine, University of Padua); Daniela Campion, Alessandro Risso, Giorgio M Saracco (Division of Gastroenterology and Hepatology, Città della Salute e della Scienza Hospital, University of Turin); Daniela Maiorca, Agostino Rizzotto (Department of Clinical and Experimental Medicine, University of Catania); Arianna Lanzi, Elga Neri, Anna Visani (Internal Medicine, Hospital of Faenza, AUSL of Romagna); Antonio Mastroianni (Internal Medicine, Hospital of Bentivoglio, AUSL of Bologna); Alberto B Alberti, Chiara Mazzarelli, Marcello Vangeli (Liver Unit, Department of Hepatology and Gastroenterology, Niguarda Hospital, Milan); Marco Marzioni, Francesca Capretti, Alba Kostandini (Department of Gastroenterology, PolItechnic University of Marche, Ancona); Giulia Magini, Maria Colpani (Gastroenterology and Transplant Hepatology, Papa Giovanni XXIII Hospital, Bergamo); Giacomo Laffi, Tommaso Gabbani, Maria Marsico (Department of Experimental and Clinical Medicine, University of Florence); Marianna Zappimbulso, Josè Petruzzi (Division of Gastroenterology, National Institute of Gastroenterology S De Bellis, Castellana Grotte); Vincenza Calvaruso (Unit of Gastroenterology and Hepatology, Biomedical Department of Internal and Specialistic Medicine, University of Palermo); Giovanni Parrella (AORN dei Colli-“Cotugno” Hospital of Naples); Nicola Caporaso, Francesco Auriemma, Maria Guarino (Department of Clinical Medicine and Surgery, Gastroenterology Unit, Federico II University of Naples); Fabio Pugliese (Internal Medicine, Department of Medical Area, University of Udine); Antonio Gasbarrini (Gastroenterology, Gemelli Foundation, Cattolica University, Rome); Pietro Leo (Gastroenterology Unit, Hospital of Cosenza);

Francesco De Leonardis, Alessandra Pecchioli, Piera Rossi (Liver Unit, Tor Vergata University, Rome); Giovanni Raimondo (Division of Clinical and Molecular Hepatology, University Hospital of Messina); Elisa Negri (Infectious Diseases and Hepatology, University Hospital of Parma, Italy); Marcello Dallio, Carmelina Loguercio (University of Campania Luigi Vanvitelli, Naples); Dario Conte (Gastroenterology and Endoscopy Unit, Foundation IRCSS Ca' Granda Ospedale Maggiore Policlinico, Milan); Natascia Celli (AUSL of Romagna, Internal Medicine, Hospital of Rimini); Roberto Bringiotti, Nicola Maurizio Castellaneta (Section of Gastroenterology, Department of Emergency and Organ Transplantation, University of Bari); Francesco Salerno (Internal Medicine, IRCSS Policlinico San Donato, University of Milan)

\section{Declaration of interests}

$\mathrm{GZ}$ is part of the speakers' bureau for Octapharma. MBe is part of the speakers' bureau for Grifols SA, Octapharma AG, Baxalta, CLS Behring $\mathrm{GmbH}$, and PPTA, and is a consultant for Baxalta and Grifols SA. MRe is part of the speakers' bureau for Grifols, Biotest, and Kedrion Pharma, and is on the advisory board for Kedrion Pharma. MT and OR are part of speakers' bureau for Baxalta. PA is part of the speakers' bureau for Baxalta and Kedrion Biopharma. PC is part of the speakers' bureau for Grifols SA, Octapharma AG, Baxalta, and Kedrion Biopharma, is consultant for Kedrion Biopharma, is on the advisory board for Grifols SA, and has a research grant from Octapharma AG. PT is part of the speakers' bureau for Grifols and Kedrion Biopharma. $\mathrm{SF}$ is part of the speakers' bureau for Grifols and Kedrion Biopharma. All other authors have no competing interests.

\section{Acknowledgments}

The trial was funded by the competitive peer-reviewed grant FARM6P824B from the Italian Medicine Agency. We thank the Italian Association for the Study of the Liver, the Italian Society of Gastroenterology, and the Italian Association of Hospital Gastroenterologists, who endorsed this study. We thank Gennaro D'Amico for his advice on and supervision of competing risks analyses, Anne Prudence Collins for editing the English manuscript, the study patients and their relatives for their participation, and the physicians and nursing staff of the participating centres for their cooperation.

\section{References}

1 GBD 2015 Mortality and Causes of Death Collaborators. Global, regional, and national life expectancy, all-cause mortality, and cause-specific mortality for 249 causes of death, 1980-2015: a systematic analysis for the Global Burden of Disease Study 2015. Lancet 2016; 388: 1459-544.

2 GBD 2015 Disease and Injury Incidence and Prevalence Collaborators. Global, regional, and national incidence, prevalence, and years lived with disability for 310 diseases and injuries, 1990-2015: a systematic analysis for the Global Burden of Disease Study 2015. Lancet 2016; 388: 1545-602.

3 Blachier M, Leleu H, Peck-Radosavljevic M, Valla DC, Roudot-Thoraval F. The burden of liver disease in Europe: a review of available epidemiological data. J Hepatol 2013; 58: 593-608.

4 Kochanek KD, Murphy SL, Xu J, Tejada-Vera G. Deaths: final data for 2014. National Vital Statistics Reports Vol 64 no 4. Hyattsville, MD: National Center for Health Statistics, 2016.

5 D'Amico G, Garcia-Tsao G, Pagliaro L. Natural history and prognostic indicators of survival in cirrhosis: a systematic review of 118 studies. J Hepatol 2006; 44: 217-31.

6 Stepanova M, De Avila L, Afendy M, et al. Direct and indirect economic burden of chronic liver disease in the United States. Clin Gastroenterol Hepatol 2017; 15: 759-66.

7 Ginés P, Quintero E, Arroyo V, et al. Compensated cirrhosis: natural history and prognostic factors. Hepatology 1987; 7: 122-28.

8 Henriksen JH, Stage JG, Schlichting P, Winkler K. Intraperitoneal pressure: ascitic fluid and splanchnic vascular pressures, and their role in prevention and formation of ascites. Scand J Clin Lab Invest 1980; 40: 493-501.

9 Wilkinson P, Sherlock S. The effect of repeated albumin infusions in patients with cirrhosis. Lancet 1962; 2: 1125-29.

10 Schrier RW, Arroyo V, Bernardi M, Epstein M, Henriksen JH, Rodés J. Peripheral arterial vasodilation hypothesis: a proposal for the initiation of renal sodium and water retention in cirrhosis. Hepatology 1988; 8: 1151-57.

11 Bernardi M, Moreau R, Angeli P, Schnabl B, Arroyo V. Mechanisms of decompensation and organ failure in cirrhosis: From peripheral arterial vasodilation to systemic inflammation hypothesis. J Hepatol 2015; 63: 1272-84.

12 Garcia-Martinez R, Caraceni P, Bernardi M, Ginés P, Arroyo V, Jalan R. Albumin: pathophysiologic basis of its role in the treatment of cirrhosis and its complications. Hepatology 2013; 58: $1836-46$.

13 Gentilini P, Casini-Raggi V, Di Fiore G, et al. Albumin improves the response to diuretics in patients with cirrhosis and ascites: results of a randomized, controlled trial. J Hepatol 1999; 30: 639-45.

14 Romanelli RG, La Villa G, Barletta G, et al. Long-term albumin infusion improves survival in patients with cirrhosis and ascites: an unblinded randomized trial. World J Gastroenterol 2006; 12: 1403-07.

15 European Association for the Study of the Liver. EASL clinical practice guidelines on the management of ascites, spontaneous bacterial peritonitis, and hepatorenal syndrome in cirrhosis. J Hepatol 2010; 53: 397-417.

16 Runyon BA, AASLD. Introduction to the revised American Association for the Study of Liver Diseases Practice Guideline management of adult patients with ascites due to cirrhosis 2012. Hepatology 2013; 57: 1651-53.

17 Moher D, Hopewell S, Schulz KF, et al. CONSORT 2010 explanation and elaboration: updated guidelines for reporting parallel group randomised trials. BMJ 2010; 340: c869.

18 Higgins JP, Altman DG, Gøtzsche PC, et al. The Cochrane Collaboration's tool for assessing risk of bias in randomised trials. BMJ 2011; 343: d5928. 
19 Moore KP, Wong F, Ginés P, et al. The management of ascites in cirrhosis: report on the consensus conference of the International Ascites Club. Hepatology 2003; 38: 258-66.

20 Szende A, Janssen B, Cabases J, eds. Self-reported population health: an international perspective based on EQ-5D. Dordrecht: Springer, 2014.

21 Scalone L, Cortesi PA, Ciampichini R, et al. Italian population-based values of EQ-5D health states. Value Health 2013; 16: 814-22.

22 Wong F, Gines P, Watson H, et al. Effects of a selective vasopressin V2 receptor antagonist, satavaptan, on ascites recurrence after paracentesis in patients with cirrhosis. J Hepatol 2010; 53: 283-90.

23 Schemper M, Smith TL. A note on quantifying follow-up in studies of failure time. Control Clin Trials 1996; 17: 343-46.

24 Pintilie, M. Competing risks: a practical perspective. Chichester: Wiley, 2006.

25 McCabe C, Claxton K, Culyer AJ. The NICE cost-effectiveness threshold: what it is and what that means. Pharmacoeconomics 2008 26: 733-44.

26 Campbell MK, Torgerson DJ. Bootstrapping: estimating confidence intervals for cost-effectiveness ratios. QJM 1999; 92: 177-82.

27 Fernández J, Navasa M, Gómez J, et al. Bacterial infections in cirrhosis: epidemiological changes with invasive procedures and norfloxacin prophylaxis. Hepatology 2002; 35: 140-48

28 Fernández J, Acevedo J, Prado V, et al. Clinical course and short-term mortality of cirrhotic patients with infections other than spontaneous bacterial peritonitis. Liver Int 2017; 37: 385-95.
29 Kunkel HG, Labby DH, Ahrens EH Jr, Shank RE, Hoagland CL. The use of concentrated human serum albumin in the treatment of cirrhosis of the liver. J Clin Invest 1948; 27: 305-19.

30 Faloon WW, Eckhardt RD, Murphy TL, Cooper AM, Davidson CS. An evaluation of human serum albumin in the treatment of cirrhosis of the liver. J Clin Invest 1949; 28: 583-94.

31 Kim SB, Chi HS, Park JS, Hong CD, Yang WS. Effect of increasing serum albumin on plasma D-dimer, von Willebrand factor, and platelet aggregation in CAPD patients. Am J Kidney Dis 1999; 33: 312-17.

32 Scalone L, Fagiuoli S, Ciampichini R, et al. The societal burden of chronic liver diseases: results from the COME study. BMJ Open Gastroenterol 2015; 2: e000025.

33 Ford I, Norrie J. Pragmatic trials. N Engl J Med 2016; 375: 454-63.

34 Moeller C, Fleischmann C, Thomas-Rueddel D, et al. How safe is gelatin? A systematic review and meta-analysis of gelatin-containing plasma expanders vs crystalloids and albumin. J Crit Care 2016; 35: 75-83.

35 Levi M, de Jonge E. Clinical relevance of the effects of plasma expanders on coagulation. Semin Thromb Hemost 2007; 33: 810-15.

36 Shaw AD, Kellum JA. The risk of AKI in patients treated with intravenous solutions containing hydroxyethyl starch. Clin J Am Soc Nephrol 2013; 8: 497-503. 